EPJ Web of Conferences 47, 06003 (2013)

DOI: $10.1051 /$ epjconf/20134706003

(C) Owned by the authors, published by EDP Sciences, 2013

\title{
Measuring ultracool properties from the UKIDSS Large Area Survey
}

\author{
Avril Day-Jones ${ }^{1,2, a}$, Federico Marocco², David Pinfield² ${ }^{2}$, Zenghua Zhang ${ }^{2}$, \\ Ben Burningham ${ }^{2}$, Nial Deacon ${ }^{3}$, Maria-Teresa Ruiz ${ }^{1}$, Jose Gallardo ${ }^{4}$, \\ Hugh Jones ${ }^{2}$, Phil Lucas ${ }^{2}$, James Jenkins ${ }^{1}$, Joana Gomes ${ }^{2}$, Stuart Folkes ${ }^{5,2}$ \\ and James Clarke ${ }^{5,2}$ \\ ${ }^{1}$ Departamento de Astronomia, Universidad de Chile, Camino el Observatorio 1515, \\ Santiago, Chile \\ ${ }^{2}$ Centre for Astrophysics Research, University of Hertfordshire, College Lane, Hatfield, \\ Hertfordshire, UK \\ ${ }^{3}$ Max Planck Institute for Astronomy, Knigstuhl, 17. 69117 Heidelberg, Germany \\ ${ }^{4}$ ALMA, Also de Cordova 3107, Vitacura, Santiago, Chile \\ ${ }^{5}$ Departamento de Fiscica y Astronomia, Facultad de Ciencias, Universidad de Valparaiso, \\ Av. Gran Bretana 1111, Valparaiso, Chile
}

\begin{abstract}
We discuss the properties and of ultracool and brown dwarfs that can be measured from current large area surveys and how fundamental parameters, such as the mass function and formation history can be measured, describing our own first measurement of the formation history in the sub-stellar regime using data from the UKIDSS Large Area Survey.
\end{abstract}

\section{INTRODUCTION}

The rise of large near infrared area surveys such as the Two Micron All Sky Survey (2MASS) and the UK Infrared Deep Sky Survey (UKIDSS) in the last two decades has increased vastly the number of brown dwarfs identified and has revealed a wide variety of physical properties and characeristics across the ultracool temperature regime. Properties such as $T_{\text {eff }}$ and $\log _{g}$ can be measured for individual brown dwarfs, however their corresponding mass, age and metallicity remain undefined due to the the limitations of ultracool model atmospheres, brought about by the lack of a unique mass-luminosity relationship for brown dwarfs. This means that the luminosity function and $T_{\text {eff }}$ distributions of field brown dwarfs depend not only on the mass function, but also on their formation history [1]. Such fundamental properties as the mass and initial mass functions, and the formation history (or birth rate) of the brown dwarf population, however requires large numbers of identified brown dwarfs. While 2MASS played an excellent starting role, discovering the first few hundred L dwarfs and the first early T dwarfs, it did not probe deep enough to reveal later type $T$ dwarfs with temperatures $<1000 \mathrm{~K}$. The UKIDSS Large Area Survey has provided the first opportunity to look at the ultracool population down to $\sim 500 \mathrm{~K}$ and has given a chance to observationally measure the formation history of the ultracool population. This contribution briefly outlines our efforts to use the UKIDSS Large Area Survey to empirically constrain the Galactic brown dwarf birth rate.

\footnotetext{
ae-mail: adjones@das.uchile.cl
}

This is an Open Access article distributed under the terms of the Creative Commons Attribution License 2.0, which permits unrestricted use, distribution, and reproduction in any medium, provided the original work is properly cited. 


\section{EPJ Web of Conferences}

\section{SIMULATIONS OF THE FORMATION HISTORY}

Simulations of the effect of how the birth rate have been performed by several authors. [1] made simulations based on just a flat birth rate and an exponential form. [2] considered a wider range of birth rates, in addition, an "empirical" birth rate, the same as measured for stars [3], representing "bursts" of formation. He also considers a "cluster" birth rate which assumes a flat, but stochastic formation, and a "halo" type birth rate, that includes formation within a 1 Gyr burst, 9 Gyr in the past. More recent simulations performed by [4] looked more specifically UKIDSS L and T dwarfs. Producing simulations that take into account several IMFs including a flat, log normal and different power laws $(\alpha=+1.0$, 0 , and -1.0 ), combined with different exponential forms of the birth rate, similar to those described above. These simulations also included the effect of Galactic disc heating, which had not been included in previous simulations of the birth rate. These simulations (see their figs 10 and 3, respectively) show that the $1100-1500 \mathrm{~K}$, corresponding to the mid-L to mid-T spectral range is most sensitive to differing birth rates.

\section{SAMPLE SELECTION}

We select a sample of mid L- mid T dwarfs from the UKIDSS LAS (DR7), with dec $\leq 20$ degrees, $J<18.1$ and $Y-J>0.8$ following the criteria of [6], who demonstrate that using $Y-J>0.8$ is largely free of M dwarfs while including all L dwarfs. We also used standard quality flags from [5] to ensure objects were point sources. Candidates were cross-matched against SDSS (DR7) to check for optical counterparts and selected if their NIR-optical colors were consistent with $\geq \mathrm{L} 4$, based on the selections of [7], using the following criteria: $J \leq 18.1, Y-J \geq 0.8, z-J \geq 2.4$ and $(J-K \geq 1.0$ or no $K$ detection) OR $z-J \geq 2.9$ and ( $J-K<1.0$ or no $K$ detection). We then removed objects with the following: $i-z<2.0$ and $\sigma(i-z)<0.35, i-J<4.7$ and $\sigma(i-j)<0.2, z-K<3.5$ and $J-K>1.0$ and $\sigma(z-K)<0.2$. As mid-T dwarfs typically have $z^{\prime}-J>3.0$ (e.g.[8]) some objects will be too faint for detection in SDSS, these were included as SDSS non-detections. We also cross matched our sample with known L and T dwarfs in dwarfarchives and retain those that have spectral types of $\geq$ L4, giving a total sample size of $\sim 320$ from $2000 \mathrm{deg}^{2}$ of UKIDSS LAS (DR7) sky.

\section{OBSERVATIONS}

Spectroscopic observations of candidates from a sub-sample, were obtained with X-shooter on the Very Large Telescope, covering the wavelength range 300-2500nm,using all three arms of X-shooter. We used a slit width of 0.9 arcsec for the VIS and NIR arms (550-1000nm and 1000-2500nm, respectively), taking integrations in an ABBA pattern, with integration times of 800 to 2000s, according to $J$ band brightness $(16.0<J<18.1)$. The data were reduced using the ESO X-shooter pipeline (version 1.3.7) to perform bias or dark subtraction, flat fielding, sky removal and extraction and merging of the orders. Telluric correction and flux calibration was done using custom written IDL routines. Finally, each spectrum was visually inspected and then binned (in the $\lambda$ direction) by 40 times to produce an average $\mathrm{SNR}=30(\mathrm{R}=880$ and 510 in the VIS and NIR arms, respectively.

\subsection{Spectroscopy}

Spectral types were determined via spectral fitting with template objects. Template spectra were obtained from the SpeX Prism Spectral Library ${ }^{1}$. Our observed spectra were then re-sampled to the same resolution as the SpeX data and spectral types were determined from the best chi-squared fit to the templates using the spectral typing scheme from [9] and [10], for L and T dwarfs, respectively.

\footnotetext{
${ }^{1}$ http://pono.ucsd.edu/adam/browndwarfs/spexprism/
} 


\section{Hot Planets and Cool Stars}

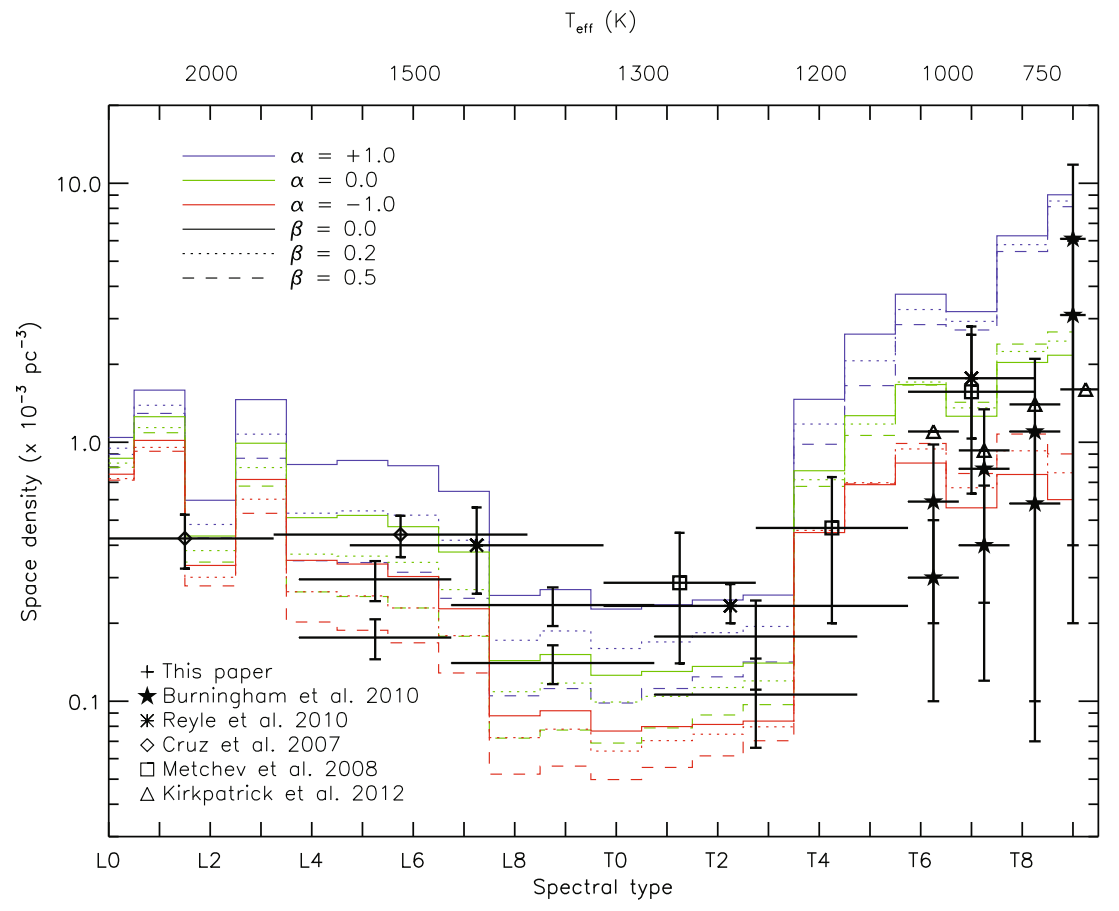

Figure 1. Our sub-sample of mid L-mid T dwarfs, overplotted with simulations from [4] with $\alpha=+1.0,0.0,-1.0$ and $\beta=0.0,0.2,0.5\left(\right.$ where $\left.\psi(M) \propto M^{-\alpha}\right)$. Upper points represent a binary fraction of $5 \%$ and lower $45 \%$.

\subsection{Unresolved binarity}

The L-T transition region is thought to be hindered with a substantial number of unresolved binaries (up to $45 \%$; [11]). We selected potential binary candidates using the criteria defined in [12] who use index-index and index-spectral type combinations to segregate possible unresolved L-T transition binaries, using known double objects as reference (see fig. 4 of [12]). We identified 12 unresolved binary candidates in this way, which were investigated further using synthetic binary template fitting. Synthetic binaries were created from standard spectral type templates from the SpeX-Prism library. These were normalized to 1 at $1.28 \mu \mathrm{m}$ and scaled to an absolute flux level, using the absolute $J$ magnitude - spectral type relation derived in [13]. Finally all the templates were combined to make our own set of synthetic unresolved binaries. We then performed a chi-squared fit of our spectra to the templates. Seven of the 12 candidates gave a better fit to a combined template. We note however that the real nature of these objects must be investigated further to confirm their true binary nature.

\section{THE BROWN DWARF FORMATION HISTORY}

We present the findings from our first sub-sample of 76 mid L to mid $\mathrm{T}$ dwarfs that occupy a complete area of $495 \mathrm{sq}$ degrees of sky $(\mathrm{RA}=22$ to $4 \mathrm{hr}$ and $\mathrm{DEC}=-2$ to $16 \mathrm{deg}$ ) to a magnitude limit of $\mathrm{J}=18.1$, by constructing a space density vs spectral type histogram. We firstly calculated the space density of our targets by converting their spectral types into effective temperatures using the $T_{\text {eff }}-$ NIR spectral type relation from [14, equation 3]. Using bin sizes of 1700-1450K, 1150-1300K and 1300-1450K (corresponding to bins of L4-L6, L7-T0 and T1-T4), we calculated the maximum distance at which an object could have been selected using the $M_{J}-$ NIR spectral type relation from [13]. With this distance limit we then calculated the volume sampled by each $T_{\text {eff }}$ bin. The derived space 
densities were then corrected for Malmquist and Eddington bias following the approach described in [8]. We combine our calculated space densities, taking into account the contribution from unresolved binaries, with those those from [5] for late-type T dwarfs, which we choose to use in favor of others available in the literature (e.g. [15], [16], [17], [18]) as this is the only space density that can be used in direct comparison to our own sample, as we probe the same magnitude range, use the same $\mathrm{M}_{J^{-}}$ spectral type relations as well as apply the same binary fractions. Our space densities are also shown as a function of spectral type in Fig. 1, where we show two points for each of our spectral type bin. Upper points represent a binary fraction of just 5\%, while lower points are for a binary fraction of $45 \%$. Also overplotted are space densities published for $\mathrm{L}$ and $\mathrm{T}$ dwarfs from the above mentioned authors. In addition we show (overplotted) the results of numerical simulations computed assuming different IMF and birth rates. Details of the simulations are presented in [4].

\section{CONCLUSIONS}

Our space densities are in general agreement (within our uncertainties) with mass functions previously measured and discussed earlier. The differences can be accounted for in large due to the use of different $\mathrm{M}_{J}$-SpT conversions by the different groups. Our space densities are however suggestive of a preference for an $\alpha<-1.0$, which is consistent with the studies of late T dwarfs alone from [8] and [5], where they conservatively find $\alpha<0$. Indeed all three samples are indicative of an $-1<\alpha<0$. It can be seen however that it is not currently possible to place robust constraints on the birth rate with this sub-sample. One of the largest sources of uncertainties is the binary fraction.

ADJ, JJ and MTR acknowledge the support of the grant CONICYT and partial support from the Center for Astrophysics FONDAP and Proyecto Basal PB06 (CATA). JJ is also supported by a FONDECYT postoctorado fellowship (3110004) and partial support by the Joint Committee ESO-Government of Chile. JG is supported by RoPACS, a Marie Curie Initial Training Network funded by the European Commission's Seventh Framework Programme.

\section{References}

[1] Chabrier G., 2002, ApJ, 567, 304

[2] Buragsser A.J., 2004, ApJ, 155, 191

[3] Rocha-Pinto H.J., et al., 2000, A\&A, 358, 869

[4] Deacon N.R., Hambly N.C., 2006, MNRAS, 371, 1722

[5] Burningham B, et al., 2010a, MNRAS, 406, 1885

[6] Hewett P.C., et al., 2006, MNRAS, 367, 454

[7] Schmidt S.J., West A.A., Hawley S.L., Pineda J.S., 2010, AJ, 139, 1808

[8] Pinfield D.J., et al., 2008, MNRAS, 390, 304

[9] Kirkpatrick J.D., et al., 1999, ApJ, 519, 802

[10] Burgasser A.J., et al., 2006, ApJ, 637, 1067

[11] Maxted P.F.L., Jeffries R.D., 2005, MNRAS, 362, 45

[12] Burgasser A.J., et al., 2010, ApJ, 710, 1142

[13] Marocco F., et al., 2010, A\&A, 524, 38

[14] Stephens D.C., et al., 2009, ApJ, 702, 154

[15] Cruz K.L., et al., 2007, AJ, 133, 439

[16] Metchev S.A., et al., 2008, ApJ, 676, 1281

[17] Reylé, C., et al., 2010, A\&A, 522, 112

[18] Kirkpatrick J.D., et al., 2012, ApJ, 753, 156 